\title{
Tissue-engineered anterior segment eye cultures maintain intraocular pressure within a normal range
}

Susannah Waxman ${ }^{1}$, Alicja Strzalkowska ${ }^{2}$, Chao Wang ${ }^{1,2,3}$, Ralitsa Loewen ${ }^{1,2}$, Yalong Dang ${ }^{1,2,4}$, Nils A. Loewen ${ }^{1,2 *}$

*Corresponding Author: Nils A. Loewen, MD, PhD

ORCID: 0000-0001-7167-1213

Department of Ophthalmology, University of Würzburg

Josef-Schneider-Straße 11

97080 Würzburg, Germany

Email: loewen.nils@gmail.com

Phone: +49-931-20120351, +49-931-20120245

\section{Author affiliations:}

1: University of Pittsburgh School of Medicine, Department of Ophthalmology, Pittsburgh, Pennsylvania, United States

2: University of Würzburg, Department of Ophthalmology, Würzburg, Germany

3: Eye Center of Xiangya Hospital, Central South University, Changsha, Hunan, China

4: Sanmenxia Central Hospital, Sanmenxia, Henan, China

Grant information: National Eye Institute K08EY022737 (NAL); Initiative to Cure Glaucoma of the Eye and Ear Foundation of Pittsburgh (NAL); Wiegand Fellowship of the Eye and Ear Foundation of Pittsburgh (YD); P30-EY08098 (NAL); Department grant by Research to Prevent Blindness (NAL); an unrestricted fellowship grant from the Xiangya Hospital of Central South University (CW).

Glaucoma is a blinding disease largely caused by increased resistance to drainage of fluid from the eye's anterior chamber, resulting in elevated intraocular pressure (IOP). A major site of fluid outflow regulation and pathology is the trabecular meshwork (TM) at the entrance of the eye's drainage system. We aimed to characterize the structural and functional properties of a newly developed tissue-engineered anterior segment eye culture model. We hypothesized that the repopulation of a decellularized TM with non-native TM cells could restore aspects of normal 
TM. The decellularization protocol removed all cells and debris while preserving the ECM. Seeded cells localized to the TM region and progressively infiltrated the meshwork ECM. Cells reached a distribution comparable to control TM after four days of perfusion culture. After a perfusion rate increase challenge, tissue-engineered cultures reestablished normal IOPs (reseeded $=13.7 \pm 0.4 \mathrm{mmHg}$, decellularized $=35.2 \pm 2.2 \mathrm{mmHg}, \mathrm{p}<0.0001$ ). EGFP expressing CrFK control cells caused a high and unstable IOP $(27.0 \pm 6.2 \mathrm{mmHg})$. In conclusion, we describe a readily available, storable, and biocompatible scaffold for anterior segment perfusion culture of non-native cells. Tissue-engineered organs demonstrated similarities to native tissues and may reduce the need for scarce donor globes in outflow research. 


\section{Introduction}

Glaucoma is a progressive optic neuropathy that affects over 70 million people worldwide and can cause irreversible blindness ${ }^{1}$. Clear fluid in the eye's anterior chamber, called aqueous humor, is produced within the confines of the globe at 2 to $3 \mu \mathrm{L} / \mathrm{min}^{2,3}$. In healthy eyes, aqueous humor outflow is in equilibrium with production but in primary open angle glaucoma, an increased outflow resistance elevates the intraocular pressure (IOP $)^{4,5}$. Intentionally elevating IOP in primate studies leads to glaucoma, while lowering it prevents it $^{6}$. IOP remains the only clinically relevant factor that can be altered to reduce glaucomatous retinal ganglion cell death and vision loss ${ }^{7,8}$. As a primary site of outflow pathogenesis, the trabecular meshwork (TM) is a target of great therapeutic interest. The TM is a dynamic, multilayer, filter-like structure that responds to environmental signals like mechanical strain and shear forces with cytoskeleton and extracellular matrix (ECM) changes to maintain a normal IOP ${ }^{9-13}$.

Simple in vitro TM cultures lack a pressure gradient and flow, defining features of glaucoma. In vitro TM perfusion models that use thin, layered scaffolds have been constructed to address these shortcomings ${ }^{14,15}$. However, these models can still not replicate the complex 3D structure of ECM beams and fibers of native TM. Their translational impact is often limited by cell physiology that is different compared to a complex organotypic substrate which strongly resembles the normal in vivo physiology ${ }^{16-19}$. Ex vivo perfused anterior segment models from a range of species have been used to examine and manipulate the TM function ${ }^{20-26}$ because there is a scarcity of whole human donor eyes for research. In contrast, TM cells from corneal rims used for corneal transplantation, TM-removal surgeries, and TM cell lines, are more readily available $e^{27,28}$ and could lend themselves to scalable studies when seeded into decellularized anterior segment scaffolds from pig eyes. As done in other tissues in research ${ }^{29-34}$ and medical applications $^{35-38}$, removal of cells from ECM can provide a bioartificial scaffold for recellularization with cells of choice. We had previously described a freeze-thaw protocol for this purpose ${ }^{39}$, but in pilot experiments detected occasional nuclear debris when we developed a protocol for recellularization. The surfactant sodium dodecyl sulfate (SDS) has been used to remove cellular material in various ECM-rich tissues, while hallmark TM ECM components like collagen, elastin, and laminin, were mostly well conserved ${ }^{29,40-42}$.

We hypothesized that decellularized porcine anterior segments ${ }^{39}$ can be repopulated with non-native TM cells to maintain IOP within a physiologic range. We seeded the eyes with porcine TM cells to eliminate species differences in this feasibility study and to obtain fresh cells with high viability. The porcine anterior segment scaffolds described here are accessible, storable, biocompatible, free of porcine cells, and can be seeded with transplanted cells. 


\section{Results}

\section{Scaffold production}

After cycles of freeze-thaw and perfusion of surfactant (FT+PS, Fig. 1a, 1a'), no cells or nuclear debris could be detected by DAPI staining (Fig 1a"). The ECM structure was well-preserved. After FT and agitation in surfactant (FT+AS, (Fig. 1b, 1b')), some remnants of nuclear debris could be made out by DAPI staining in the TM most distal from the anterior chamber (Fig. 1b"). FT+AS samples on days 1, 2, and 5 days looked similar. FT alone (Fig. 1c) destroyed cells but could show displaced nuclear material in the mid and distal TM (Fig. 1c' and c'). Untreated controls (Fig. 1d) had a normal TM cellularity (Fig. 1d') and an even DAPI staining pattern. Here, DAPI was limited to nuclei within cells instead of the diffuse staining in FT+AS ( $\left.b^{\prime \prime}\right)$ and FT ( $\left.c^{\prime \prime}\right)$. Mean DAPI fluorescence in FT+PS was much lower than in controls $(p=0.010)$. Despite a different histological appearance as described, the mean DAPI fluorescence intensity was relatively similar in FT+AS ( $\left.b^{\prime \prime}\right), F T\left(c^{\prime \prime}\right)$ and controls ( $d^{\prime \prime}, F T+A S$ vs controls: $p=0.575, F T$ vs controls: $p=0.387$ ). Because FT+PS had a well-preserved ECM but were devoid of cells or nuclear debris, we used FT+PS scaffolds for all subsequent experiments.

IOP remained within a normal range throughout the perfusion-decellularization process without evidence of any abnormal physical stress on the ECM (Fig. 2, n = 15). After 24 hours of washing, scaffold IOP remained stable (within a $0.6 \mathrm{mmHg}$ range) through the remainder of the process. Washed scaffolds appeared grossly normal. 24 scaffolds were produced. Eight IOP recordings were lost due to hardware error. One eye was contaminated and not included in the IOP analysis.

\section{Scaffold repopulation and IOP}

We transduced robust CrFK cells with an eGFP expressing lentiviral FIV vector, enriched them by fluorescence-activated cell sorting (FACS), and seeded them onto scaffolds to establish protocols for repopulation. The fluorescent signal of eGFP-expressing CrFK cells could be visualized at all time-points $(24,48$, and 144 hours) and localized in the TM region. Occasional fluorescent cells could be seen on the corneal endothelium at 24 hours but not at subsequent time-points. Fluorescence was at times seen where the ciliary body had been attached. The TM was significantly brighter than other regions by an average of $90.1 \pm 7.5 \%(p<0.001$, Fig. 3 ).

The depth of cell infiltration into the TM was measured and compared to control at each time-point (supplemental material 1). The average depth of cells in the TM increased over time and peaked day four (Fig. 4, Table 1). CrFK cell depth at 24 and 48 hours was significantly lower than control $(p<0.05)$ while the depth of TM cells at 96 and CrFK cells at 144 hours was not significantly different $(p=0.19,0.06)$. At 24 hours post-seeding, some cells could be seen histologically on the corneal endothelium, matching occasional cells fluorescing in Fig. 3. Cells migrated in the direction of outflow over time. Additionally, cellularity increased with time (Table 1, n= 3458 nuclei measured total). After 96 hours in culture, many cells had large nuclei with euchromatin, indicating active transcription during infiltration. No cells were seen in the 
region of the angular aqueous plexus, a region similar to Schlemm's canal and proximal collector channels in primates ${ }^{43}$.

A stable IOP baseline was achieved after 24 hours (Fig. 5). No significant difference was found between decellularized (D) and scaffolds reseeded (RS) with TM cells at baseline ( $D=$ $11.8 \pm 0.5 \mathrm{mmHg}, \mathrm{RS}=12.4 \pm 0.5 \mathrm{mmHg}, \mathrm{p}=0.40)$. There was no difference between $\mathrm{D}$ and $\mathrm{RS}$ after seeding group $\mathrm{RS}(\mathrm{D}=9.2 \pm 0.4 \mathrm{mmHg}, \mathrm{RS}=8.5 \pm 0.2 \mathrm{mmHg}, \mathrm{p}=0.07)$. In contrast, scaffolds seeded with CrFK cells had a higher IOP that was quite unstable as indicated by a large standard deviation as a measure of the amount of variation $(27.0 \pm 17.3 \mathrm{mmHg}(\mathrm{avg} \pm \mathrm{SD})$ ) when compared to TM cells $(8.5 \pm 2.7 \mathrm{mmHg}(\operatorname{avg} \pm \mathrm{SD})$ ). At 48 hours post TM reseeding in $\mathrm{RS}$ or sham procedure in $D$, the infusion rate was doubled from 3 to $6 \mu \mathrm{L} / \mathrm{min}$ to challenge the TM's IOP maintenance response. One normal control eye developed a leak and had to be removed, reducing the count to $n=7$. RS maintained IOP within a normal range that was not different from normal control eyes $(p>0.05, n=8$ ), while $D$ became hypertensive ( $R S=13.7 \pm 0.4 \mathrm{mmHg}, n=8, D=35.2 \pm 2.2$ $\mathrm{mmHg}, \mathrm{p}<0.0001, \mathrm{n}=8$, Fig. 5). The IOPs of RS and $\mathrm{D}$ after challenge were significantly different from their respective IOPs in the reseed/sham phase (both $p<0.001$, average increases of 8.05 and $25.04 \mathrm{mmHg}$, respectively). The IOP of normal control eyes after the challenge was also significantly higher than before the challenge $(p<0.001)$. The IOP of challenged RS was not different from baseline $(p=0.06)$. Neither RS nor normal control eyes showed a homeostatic IOP decline ${ }^{11}$.

\section{Discussion}

In this study, we developed a tissue-engineered anterior segment model that has an outflow function with similarities to standard ex vivo and in vivo models. After an infusion rate challenge, anterior segments with repopulated TM maintained a physiological IOP while anterior segments with decellularized TM nearly tripled. Our results were similar to those observed in standard human anterior segment perfusion models with infusion rate doubling ${ }^{11}$ and after IOP increase ${ }^{44}$.

TM cell numbers decrease gradually with age ${ }^{45,46}$. Both increased age and reduced TM cellularity are a major risk factor for the development of glaucoma. On the other hand, partially removing TM cells can also reduce IOP at least temporarily ${ }^{47}$. It has been previously shown that the presence of denuded TM beams can eventually fuse, associated with TM collapse, reduction of intertrabecular spaces for outflow, and pathologically elevated $1 \mathrm{IOP}^{48-53}$. Abu-Hassan et al. ${ }^{23}$ recently showed that the transplantation of human TM cells or TM-like iPSCs into the anterior segment perfusion model can restore outflow after killing about $1 / 3$ rd of TM cells with saponin. In our study, all resident cells were removed to guarantee that any observed effect could only be caused by the transplanted cells but not by residual ones. We also washed any debris off scaffolds and avoided saponin to allow for a healthy TM cell function. While SDS + Triton X-100-mediated decellularization was chosen for this model in part due to demonstrated conservation of ECM components such as glycosaminoglycans (GAGs), 
collagen, elastin, and laminin ${ }^{40,42,54,55}$, hampered TM cell adhesion may reflect mild GAG loss. We chose porcine TM cells for reseeding because they can be rapidly generated and have a high viability.

Even though there was no Schlemm's canal, this model allowed us to isolate the effects of TM cells on outflow physiology in the TM region. The TM has a multitude of mechanisms at its disposal to adjust outflow that includes cytoskeletal as well as extracellular matrix changes. Important mediators include PGF $2 \alpha, \operatorname{TGF} \beta, \mathrm{IL}-1 \alpha / \beta, \mathrm{TNF} \alpha$, nitric oxide, adenosine, and Rho kinase $^{9-13,56}$ among others. We focused on establishing TM ablation and repopulation and did not investigate the mechanisms of IOP regulation in this model. It is striking that only TM cells but not CrFK cells, a spontaneously immortalized kidney epithelial cell line, could maintain IOP. The increased IOP after perfusion challenge in decellularized scaffolds is likely caused by collapsing intertrabecular spaces and spaces within the angular aqueous plexus. In contrast to TM cells, CrFK cells were unable to maintain IOP within a physiological range. Instead, it was high and quite unstable as evidenced by a large standard deviation, a measure of the amount of variation. As a spontaneously immortalized epithelial kidney cell line, CrFK cells have a different migration speed and threshold for contact inhibition compared to primary TM cells. The porcine TM is nearly three times as thick as human TM. TM compression as a result of increased IOP can lead to narrowing and rarefaction of outflow pathways with a declining facility as established in bovine $^{57}$ and human eyes ${ }^{58}$. In contrast, when porcine eyes are subjected to ab interno trabeculectomy, outflow does increase, however ${ }^{59-61}$.

In the current study, fluorescent cells infiltrated the TM to a depth that was comparable to resident cells of normal controls. In pilot studies not presented above, we seeded 1 million cells, a cell number closer to that of a young, healthy $\mathrm{TM}^{46}$ but this resulted in a low tissue-engineered TM cellularity. This is consistent with cell loss percentages in other tissue-engineered organs ${ }^{29,62}$. It is possible that seeded TM cells require sufficient cell-cell contact early in the process of meshwork infiltration as they cannot survive or proliferate otherwise. Cells settled extensively along and into the TM in most quadrants but not all. This supports the notion that flow through a comprehensively ablated anterior segment is still segmental. Similar to the eGFP expressing CrFK cells in this study, outflow can be visualized with tracers that do not readily pass through the TM but typically are 10 to 100 times smaller ${ }^{59}$ or with fluorescent dyes that have a fast TM passage time and allow for precise computation of focal outflow ${ }^{59,61,63}$. Determining changes of segmental outflow more precisely with fluorescent dye canalograms will be in future studies that use this model.

Limitations of this study are the lack of additional canalograms to test for changing segmental flow patterns, confirmation of the predominant mechanism by which TM cells keep IOP within a normal range, and the use of porcine instead of human eyes that have a Schlemm's canal. Cells of the TM are a heterogeneous population of uveal, corneoscleral, juxtacanalicular, SC-like endothelial cells, and stem cells. For this reason, future characterization of TM cells in 
these scaffolds needs to include an analysis of gene expression and location to determine the similarities and differences between TE-cultures and normal anterior segments.

IOP in scaffolds with reseeded TM cells was not different from normal control eyes and remained within a normal range but this was not present in sham or CrFK seeded eyes. A homeostatic response with an IOP decline to pre-challenge levels was absent in both reseeded and normal control eyes. Our observation is different from prior publications that used gravity-driven perfusion with a fixed IOP. In those, an increasing facility was noted and termed "TM homeostasis"11,64 to contrast "washout" ${ }^{65-70}$. Perhaps an infusion rate change is more difficult to compensate than an increase in pressure in gravity-driven systems.

Lastly, losing seeded cells is a common problem. For instance, Ott et al. report that $46 \%$ of cells seeded into bioartificial heart culture were lost already within 20 minutes ${ }^{29}$. Improving efficiency, for example, through the use of polymer gels will be necessary ${ }^{71}$.

TE-ex vivo cultures provide a new tool in the aqueous humor outflow research toolkit that is easy to generate and scale. In vitro and 2D perfusion cultures are harder to produce and/or lack biofidelic cues from a physiological multilayered, 3D environment, a hallmark of this tissue. Not only is generating the scaffolds straightforward, the ability to store them at $-80^{\circ} \mathrm{C}$ and install them rapidly adds flexibility. The expansion rates of primary cells harvested clinically during ab interno glaucoma surgery can vary considerably but stored scaffolds can be deployed instantly and at a scale that cannot be matched by human donor eyes.

In conclusion, we developed a fully decellularized porcine anterior segment scaffold and reseeded it with non-native meshwork cells. Tissue-engineered ex vivo cultures demonstrated localization of transplanted cells to the TM region, cell infiltration of ECM, and IOP maintenance ability after infusion rate challenge, each hallmarks of ex vivo culture structure and function. These scaffolds may allow testing of patient-derived iPSCs, human TM cell lines, or expanded human primary TM cells with a higher throughput and reduced need for human donor eyes.

\section{Methods}

A total of 49 anterior segments were prepared as previously reported ${ }^{20,56,72}$. Briefly, porcine eyes were acquired within 4 hours of sacrifice, decontaminated with povidone-iodine, and hemisected along the equator in an aseptic biosafety cabinet. The posterior segment, lens, and iris were carefully removed.

\section{Scaffold production}

Freeze-thaw-treated ${ }^{39,73}$ anterior segments (FT) were sealed in an airtight container and cycled between $-80^{\circ} \mathrm{C}$ and room temperature two times to lyse all native cells ${ }^{39,73}$. Freeze-thawed scaffolds were perfused with culture media (DMEM supplemented with $1 \%$ FBS and $1 \mathrm{X}$ antibiotic/antimycotic) for 5 days $(n=4)$. A time-course assay was conducted to determine the minimum time to produce decellularized scaffolds via immersion/agitation-mediated decellularization (IA). Freeze-thawed anterior segments were placed in SDS solution $(0.01 \%$ 
wt/vol in PBS + anti-anti) for 1 day $(n=3), 2$ days $(n=3)$, and 5 days $(n=3)$. Following SDS incubation, solutions were changed to $0.1 \%$ TritonX-100 for 24 hours and then a perfusion culture media wash for 48 hours. Samples were affixed to a vertical stage rotated at 20 RPM during each incubation. Fluid exchanges were performed every 24 hours.

For perfusion-mediated decellularization $(P)$, we modified an existing matrix production protocol used in bioartificial heart construction ${ }^{29}$. Freeze-thawed segments were maintained via constant-rate perfusion ${ }^{21}$ at $6 \mu \mathrm{L} / \mathrm{min}$ with SDS solution for 24 hours, TritonX-100 solution for 24 hours, and perfusion culture media for 48 hours $(n=24)$. Untreated control samples were maintained for 5 days with perfusion culture media $(n=4)$. All scaffolds were stored at $-80^{\circ} \mathrm{C}$ and up to two months before reseeding. To avoid any confounding effects from prior experiments, none were reused.

\section{Cell culture}

Porcine TM culture was performed as done previously ${ }^{20,74}$. The TM was dissected away from anterior segments under an ophthalmic operating microscope (Stativ S4, Carl Zeiss, Oberkochen, Germany) and cut into $0.5 \mathrm{~mm}^{3}$ segments. Tissue pieces were cultured in T25 flasks containing OptiMEM (31985-070, Gibco, Life Technologies, Grand Island, NY, USA), supplemented with 5\% FBS and antibiotic/antimycotic (15240062, Thermo Fisher Scientific, Waltham, MA, USA). The method used here to obtain cells from the porcine angular plexus naturally provides a non-homogenous cell population. Cells were passaged at $80 \%$ confluence and used for experiments at passages 2-4.

For initial scaffold biocompatibility testing and real-time tracking of seeded cells, we used a versatile CrFK cell-line (CRFK ATCC CCL-94) transduced with eGFP-expressing feline immunodeficiency viral (FIV) vector GINSIN ${ }^{21,47}$. Cells were transduced at a multiplicity of infection (MOI) of 5 transducing units (TU) per cell and enriched by fluorescence-activated cell sorting (FACS) after 7 days with a high fluorescence gate cut-off that eliminated all non-fluorescent cells. GINSIN transduced CrFK cells were maintained in 2.5\% FBS DMEM with antibiotic and antimycotic and passaged at $80 \%$ confluence.

\section{Live-cell tracking}

Three million GINSIN transduced CrFK cells were seeded into scaffolds to determine biocompatibility and cell localization in real-time. A $20 \mathrm{G}$ cannula (BD PrecisionGlide 305176) attached to a $1 \mathrm{~mL}$ syringe (Norm-Ject Tuberkulin Luer 4010-200V0) was connected to a $3 \mathrm{~cm}$ length of perfusion tubing. The inlet and outlet of each perfusion dish were disconnected from the perfusion system and reconnected to the $1 \mathrm{~mL}$ syringes. A $200 \mu \mathrm{L}$ bolus of culture media was removed from anterior chambers through the outlet. A $200 \mu \mathrm{L}$ bolus of 3 million GINSIN-CrFK cells was slowly introduced into the anterior chamber. Cultures were gravity perfused at $15 \mathrm{mmHg}$ for 20 minutes. Dishes were positioned with corneas facing downward and perfusion culture was restarted. 
TM was visualized from the underside of transparent perfusion dishes with an epifluorescence-equipped dissecting microscope (Olympus SZX16 with GFP filter cube and DP80 monochrome/color camera; Olympus Corp., Center Valley, PA, USA) and imaged at 24, 48, and 144 hours post-seeding. Mean fluorescent intensity of images was measured in four concentric regions (central cornea (1), peripheral cornea (2), TM (3), and sclera/ciliary body remnant (4), Fig. 3, bottom right) in $\mathrm{Fiji}^{75}$ and compared. A uniform threshold was applied to fluorescence images to aid visualization in Fig. 3, right panels.

\section{IOP maintenance challenge}

Decellularized scaffolds were perfused for 24 hours with TM culture media before seeding to achieve stable baseline IOPs. Three million porcine TM cells were seeded as described above in RS $(n=8)$ while $D(n=8)$ received a sham bolus of cell-free TM culture media. Normal anterior segment cultures were used as controls $(n=8)$. After 48 hours, the infusion rate was doubled from $3 \mu \mathrm{L} / \mathrm{min}$ to $6 \mu \mathrm{L} / \mathrm{min}$ to challenge the TM's IOP maintenance response.

\section{Histology}

Samples were fixed in 4\% PFA for 48 hours, embedded in paraffin, and sectioned at $6 \mu \mathrm{m}$ thickness. Sections were stained with hematoxylin and eosin (H\&E) for morphological analysis and DAPI for DNA content. A specimen was analyzed from the center of both the superior and inferior portion of each anterior segment.

\section{Statistical analysis}

Fluorescence intensities of DAPI-labeled scaffold samples were compared to control. Live-cell eGFP fluorescence of the TM region was compared to all other locations. Cell depth at each time-point was compared to control. IOP recordings were sampled into 2-hour blocks, and D was compared to RS at each experimental phase (baseline, cell seeding, and challenge). Periods of 8 hours after media refilling were excluded from the analysis to allow for IOP stabilization. Statistical comparisons were conducted with a student's t-test in Python 3.6. Data are expressed as mean \pm SEM unless otherwise noted. P-values $<0.05$ were considered statistically significant.

\section{Acknowledgments}

This study was funded by the Initiative to Cure Glaucoma of the Eye and Ear Foundation of Pittsburgh (NAL), by NEI Grant K08EY022737, by NIH CORE Grant P30 EY08098 to the Department of Ophthalmology, and an unrestricted grant from Research to Prevent Blindness, New York, NY. 


\section{Compliance with Ethical Standards}

This article does not contain any studies with human participants. No animals were sacrificed for this study. Porcine eyes were acquired from a local abattoir; permission was granted from the owners of the animals for use of eyes in research. Approval by an ethics committee or institutional animal care and use committee was not required.

\section{Competing Interests}

The authors declare no competing interests.

\section{Data Availability}

The datasets generated and analyzed during the current study are available from the corresponding author on reasonable request.

\section{Corresponding Author}

Correspondence to Nils A. Loewen 


\section{References}

1. Tham, Y.-C. et al. Global prevalence of glaucoma and projections of glaucoma burden through 2040: a systematic review and meta-analysis. Ophthalmology 121, 2081-2090 (2014).

2. Maus, T. L. \& Brubaker, R. F. Measurement of aqueous humor flow by fluorophotometry in the presence of a dilated pupil. Invest. Ophthalmol. Vis. Sci. 40, 542-546 (1999).

3. Goel, M., Picciani, R. G., Lee, R. K. \& Bhattacharya, S. K. Aqueous humor dynamics: a review. Open Ophthalmol. J. 4, 52-59 (2010).

4. Chan, M. P. Y. et al. Glaucoma and intraocular pressure in EPIC-Norfolk Eye Study: cross sectional study. BMJ 358, j3889 (2017).

5. Sommer, A. et al. Relationship between intraocular pressure and primary open angle glaucoma among white and black Americans. The Baltimore Eye Survey. Arch. Ophthalmol. 109, 1090-1095 (1991).

6. Nickells, R. W. et al. Surgical lowering of elevated intraocular pressure in monkeys prevents progression of glaucomatous disease. Exp. Eye Res. 84, 729-736 (2007).

7. Gordon, M. O. \& Kass, M. A. The Ocular Hypertension Treatment Study: design and baseline description of the participants. Arch. Ophthalmol. 117, 573-583 (1999).

8. Leske, M. C. et al. Predictors of long-term progression in the early manifest glaucoma trial. Ophthalmology 114, 1965-1972 (2007).

9. Bradley, J. M. et al. Effects of mechanical stretching on trabecular matrix metalloproteinases. Invest. Ophthalmol. Vis. Sci. 42, 1505-1513 (2001).

10. Fleenor, D. L. et al. TGFbeta2-induced changes in human trabecular meshwork: implications for intraocular pressure. Invest. Ophthalmol. Vis. Sci. 47, 226-234 (2006).

11. Acott, T. S. et al. Intraocular pressure homeostasis: maintaining balance in a high-pressure environment. J. Ocul. Pharmacol. Ther. 30, 94-101 (2014).

12. Vranka, J. A. et al. Biomechanical Rigidity and Quantitative Proteomics Analysis of Segmental Regions of the Trabecular Meshwork at Physiologic and Elevated Pressures. Invest. Ophthalmol. Vis. Sci. 59, 246-259 (2018).

13. Zenkel, M. et al. Differential gene expression in pseudoexfoliation syndrome. Invest. Ophthalmol. Vis. Sci. 46, 3742-3752 (2005).

14. Torrejon, K. Y. et al. Bioengineered glaucomatous 3D human trabecular meshwork as an in vitro disease model. Biotechnol. Bioeng. 113, 1357-1368 (2016).

15. Torrejon, K. Y. et al. Recreating a human trabecular meshwork outflow system on microfabricated porous structures. Biotechnol. Bioeng. 110, 3205-3218 (2013).

16. Duval, K. et al. Modeling Physiological Events in 2D vs. 3D Cell Culture. Physiology 32, 266-277 (2017).

17. Kapałczyńska, M. et al. 2D and 3D cell cultures - a comparison of different types of cancer cell cultures. Arch. Med. Sci. 14, 910-919 (2018).

18. Huh, D., Hamilton, G. A. \& Ingber, D. E. From 3D cell culture to organs-on-chips. Trends Cell Biol. 21, 745-754 (2011).

19. Alford, P. W., Feinberg, A. W., Sheehy, S. P. \& Parker, K. K. Biohybrid thin films for measuring contractility in engineered cardiovascular muscle. Biomaterials 31, 3613-3621 (2010).

20. Dang, Y. et al. A porcine ex vivo model of pigmentary glaucoma. Sci. Rep. 8, 5468 (2018). 
21. Loewen, R. T. et al. A Porcine Anterior Segment Perfusion and Transduction Model With Direct Visualization of the Trabecular Meshwork. Invest. Ophthalmol. Vis. Sci. 57, 1338-1344 (2016).

22. Sherwood, J. M., Reina-Torres, E., Bertrand, J. A., Rowe, B. \& Overby, D. R. Measurement of Outflow Facility Using iPerfusion. PLoS One 11, e0150694 (2016).

23. Abu-Hassan, D. W., Li, X., Ryan, E. I., Acott, T. S. \& Kelley, M. J. Induced pluripotent stem cells restore function in a human cell loss model of open-angle glaucoma. Stem Cells 33, 751-761 (2015).

24. Loewen, N. et al. Genetic modification of human trabecular meshwork with lentiviral vectors. Hum. Gene Ther. 12, 2109-2119 (2001).

25. Fautsch, M. P. et al. Perfusion of his-tagged eukaryotic myocilin increases outflow resistance in human anterior segments in the presence of aqueous humor. Invest. Ophthalmol. Vis. Sci. 47, 213-221 (2006).

26. Loewen, N. et al. Preservation of aqueous outflow facility after second-generation FIV vector-mediated expression of marker genes in anterior segments of human eyes. Invest. Ophthalmol. Vis. Sci. 43, 3686-3690 (2002).

27. Dang, Y. et al. Outflow enhancement by three different ab interno trabeculectomy procedures in a porcine anterior segment model. Graefes Arch. Clin. Exp. Ophthalmol. 256, 1305-1312 (2018).

28. Swaminathan, S. S. et al. Histologic Analysis of Trabecular Meshwork Obtained from Kahook Dual Blade Goniotomy. Am. J. Ophthalmol. (2018) doi:10.1016/j.ajo.2018.05.028.

29. Ott, H. C. et al. Perfusion-decellularized matrix: using nature's platform to engineer a bioartificial heart. Nat. Med. 14, 213-221 (2008).

30. Guan, Y. et al. Porcine kidneys as a source of ECM scaffold for kidney regeneration. Mater. Sci. Eng. C Mater. Biol. Appl. 56, 451-456 (2015).

31. Ott, H. C. et al. Regeneration and orthotopic transplantation of a bioartificial lung. Nat. Med. 16, 927-933 (2010).

32. Song, J. J. et al. Regeneration and experimental orthotopic transplantation of a bioengineered kidney. Nat. Med. 19, 646-651 (2013).

33. Dahl, S. L. M., Koh, J., Prabhakar, V. \& Niklason, L. E. Decellularized native and engineered arterial scaffolds for transplantation. Cell Transplant. 12, 659-666 (2003).

34. Lin, P., Chan, W. C. W., Badylak, S. F. \& Bhatia, S. N. Assessing porcine liver-derived biomatrix for hepatic tissue engineering. Tissue Eng. 10, 1046-1053 (2004).

35. Macchiarini, P. et al. Clinical transplantation of a tissue-engineered airway. Lancet 372, 2023-2030 (2008).

36. Wainwright, D. J. Use of an acellular allograft dermal matrix (AlloDerm) in the management of full-thickness burns. Burns 21, 243-248 (1995).

37. Bejjani, G. K., Zabramski, J. \& Durasis Study Group. Safety and efficacy of the porcine small intestinal submucosa dural substitute: results of a prospective multicenter study and literature review. J. Neurosurg. 106, 1028-1033 (2007).

38. Eichler, C. et al. A Head to Head Comparison Between SurgiMend ${ }^{\circledR}$--Fetal Bovine Acellular Dermal Matrix and Tutomesh ${ }^{\circledR}$--A Bovine Pericardium Collagen Membrane in Breast Reconstruction in 45 Cases. In Vivo 31, 677-682 (2017).

39. Dang, Y. et al. Freeze-thaw decellularization of the trabecular meshwork in anex vivoeye perfusion model. PeerJ 5, e3629 (2017).

40. Guyette, J. P. et al. Bioengineering Human Myocardium on Native Extracellular Matrix. Circ. Res. 
118, 56-72 (2016).

41. Schaner, P. J. et al. Decellularized vein as a potential scaffold for vascular tissue engineering. J. Vasc. Surg. 40, 146-153 (2004).

42. Gerli, M. F. M., Guyette, J. P., Evangelista-Leite, D., Ghoshhajra, B. B. \& Ott, H. C. Perfusion decellularization of a human limb: A novel platform for composite tissue engineering and reconstructive surgery. PLoS One 13, e0191497 (2018).

43. Johnson, M., Johnson, D. H., Kamm, R. D., DeKater, A. W. \& Epstein, D. L. The filtration characteristics of the aqueous outflow system. Exp. Eye Res. 50, 407-418 (1990).

44. Borrás, T., Rowlette, L. L. S., Tamm, E. R., Gottanka, J. \& Epstein, D. L. Effects of elevated intraocular pressure on outflow facility and TIGR/MYOC expression in perfused human anterior segments. Invest. Ophthalmol. Vis. Sci. 43, 33-40 (2002).

45. McMenamin, P. G., Lee, W. R. \& Aitken, D. A. Age-related changes in the human outflow apparatus. Ophthalmology 93, 194-209 (1986).

46. Alvarado, J., Murphy, C., Polansky, J. \& Juster, R. Age-related changes in trabecular meshwork cellularity. Invest. Ophthalmol. Vis. Sci. 21, 714-727 (1981).

47. Zhang, Z. et al. Outflow tract ablation using a conditionally cytotoxic feline immunodeficiency viral vector. Invest. Ophthalmol. Vis. Sci. 55, 935-940 (2014).

48. Tektas, O.-Y. \& Lütjen-Drecoll, E. Structural changes of the trabecular meshwork in different kinds of glaucoma. Exp. Eye Res. 88, 769-775 (2009).

49. Roberts, F. \& Thum, C. K. Lee's Ophthalmic Histopathology. (Springer Science \& Business Media, 2013).

50. King, B. J., Burns, S. A., Sapoznik, K. A., Luo, T. \& Gast, T. J. High-Resolution, Adaptive Optics Imaging of the Human Trabecular Meshwork In Vivo. Transl. Vis. Sci. Technol. 8, 5 (2019).

51. Gong, H. \& Swain, D. L. SD The histopathological changes in the trabecular outflow pathway and their possible effects on aqueous outflow in eyes with primary open-angle glaucoma. Glaucoma research and clinical advances 2018, 17-40 (2016).

52. Tamm, E. R. Functional Morphology of the Trabecular Meshwork*. Encyclopedia of the Eye 157-163 (2010) doi:10.1016/b978-0-12-374203-2.00092-0.

53. Gottanka, J., Johnson, D. H., Grehn, F. \& Lütjen-Drecoll, E. Histologic findings in pigment dispersion syndrome and pigmentary glaucoma. J. Glaucoma 15, 142-151 (2006).

54. Mirsadraee, S. et al. Development and characterization of an acellular human pericardial matrix for tissue engineering. Tissue Eng. 12, 763-773 (2006).

55. Singelyn, J. M. et al. Naturally derived myocardial matrix as an injectable scaffold for cardiac tissue engineering. Biomaterials vol. 30 5409-5416 (2009).

56. Dang, Y. et al. RKI-1447, a Rho kinase inhibitor, causes ocular hypotension, actin stress fiber disruption, and increased phagocytosis. Graefes Arch. Clin. Exp. Ophthalmol. 257, 101-109 (2019).

57. Battista, S. A. et al. Reduction of the available area for aqueous humor outflow and increase in meshwork herniations into collector channels following acute IOP elevation in bovine eyes. Invest. Ophthalmol. Vis. Sci. 49, 5346-5352 (2008).

58. Karyotakis, N. G., Ginis, H. S., Dastiridou, A. I., Tsilimbaris, M. K. \& Pallikaris, I. G. Manometric measurement of the outflow facility in the living human eye and its dependence on intraocular pressure. Acta Ophthalmol. 93, e343-8 (2015).

59. Loewen, R. T. et al. Quantification of Focal Outflow Enhancement Using Differential Canalograms. 
Invest. Ophthalmol. Vis. Sci. 57, 2831-2838 (2016).

60. Dang, Y. et al. Rapid learning curve assessment in an ex vivo training system for microincisional glaucoma surgery. Sci. Rep. 7, 1605 (2017).

61. Loewen, R. T. et al. Regionally Discrete Aqueous Humor Outflow Quantification Using Fluorescein Canalograms. PLoS One 11, e0151754 (2016).

62. Wendt, D., Marsano, A., Jakob, M., Heberer, M. \& Martin, I. Oscillating perfusion of cell suspensions through three-dimensional scaffolds enhances cell seeding efficiency and uniformity. Biotechnol. Bioeng. 84, 205-214 (2003).

63. Parikh, H. A. et al. Differential Canalograms Detect Outflow Changes from Trabecular Micro-Bypass Stents and Ab Interno Trabeculectomy. Sci. Rep. 6, 34705 (2016).

64. Abu-Hassan, D. W., Li, X., Ryan, E. I., Acott, T. S. \& Kelley, M. J. Induced pluripotent stem cells restore function in a human cell loss model of open-angle glaucoma. Stem Cells 33, 751-761 (2015).

65. Kaufman, P. L., True-Gabelt, B. \& Erickson-Lamy, K. A. Time-dependence of perfusion outflow facility in the cynomolgus monkey. Curr. Eye Res. 7, 721-726 (1988).

66. Erickson-Lamy, K., Schroeder, A. M., Bassett-Chu, S. \& Epstein, D. L. Absence of time-dependent facility increase (washout) in the perfused enucleated human eye. Invest. Ophthalmol. Vis. Sci. 31, 2384-2388 (1990).

67. Gaasterland, D. E., Pederson, J. E. \& MacLellan, H. M. Perfusate effects upon resistance to aqueous humor outflow in the rhesus monkey eye. A comparison of glutathione-bicarbonate Ringer's solution to pooled aqueous humor as perfusate. Invest. Ophthalmol. Vis. Sci. 17, 391-397 (1978).

68. Johnson, M., Chen, A., Epstein, D. L. \& Kamm, R. D. The pressure and volume dependence of the rate of wash-out in the bovine eye. Curr. Eye Res. 10, 373-375 (1991).

69. Sit, A. J. et al. The Role of Soluble Proteins in Generating Aqueous Outflow Resistance in the Bovine and Human Eye. Experimental Eye Research vol. 64 813-821 (1997).

70. Erickson, K. A. \& Kaufman, P. L. Comparative effects of three ocular perfusates on outflow facility in the cynomolgus monkey. Curr. Eye Res. 1, 211-216 (1981).

71. Maidhof, R., Marsano, A., Lee, E. J. \& Vunjak-Novakovic, G. Perfusion seeding of channeled elastomeric scaffolds with myocytes and endothelial cells for cardiac tissue engineering. Biotechnol. Prog. 26, 565-572 (2010).

72. Waxman, S. et al. Structure-Function Changes of the Porcine Distal Outflow Tract in Response to Nitric Oxide. Invest. Ophthalmol. Vis. Sci. 59, 4886-4895 (2018).

73. Burk, J. et al. Freeze-thaw cycles enhance decellularization of large tendons. Tissue Eng. Part $C$ Methods 20, 276-284 (2014).

74. Wang, C. et al. Impact of pigment dispersion on trabecular meshwork cells. Graefes Arch. Clin. Exp. Ophthalmol. (2019) doi:10.1007/s00417-019-04300-7.

75. Schindelin, J. et al. Fiji: an open-source platform for biological-image analysis. Nat. Methods $\mathbf{9}$, 676-682 (2012). 


\section{Author Contributions}

SW: manuscript writing with input from all co-authors, data collection, data analysis, figure creation, contributed to study design. CW: experimentation, data collection, reviewed manuscript. YD: experimentation, reviewed manuscript. RL: experimentation, reviewed manuscript. NAL: experimentation, funding, study design, oversaw data collection, manuscript writing. 


\section{Figures}

\section{Figure 1: freeze-thaw + surfactant perfusion decellularized AS scaffolds}

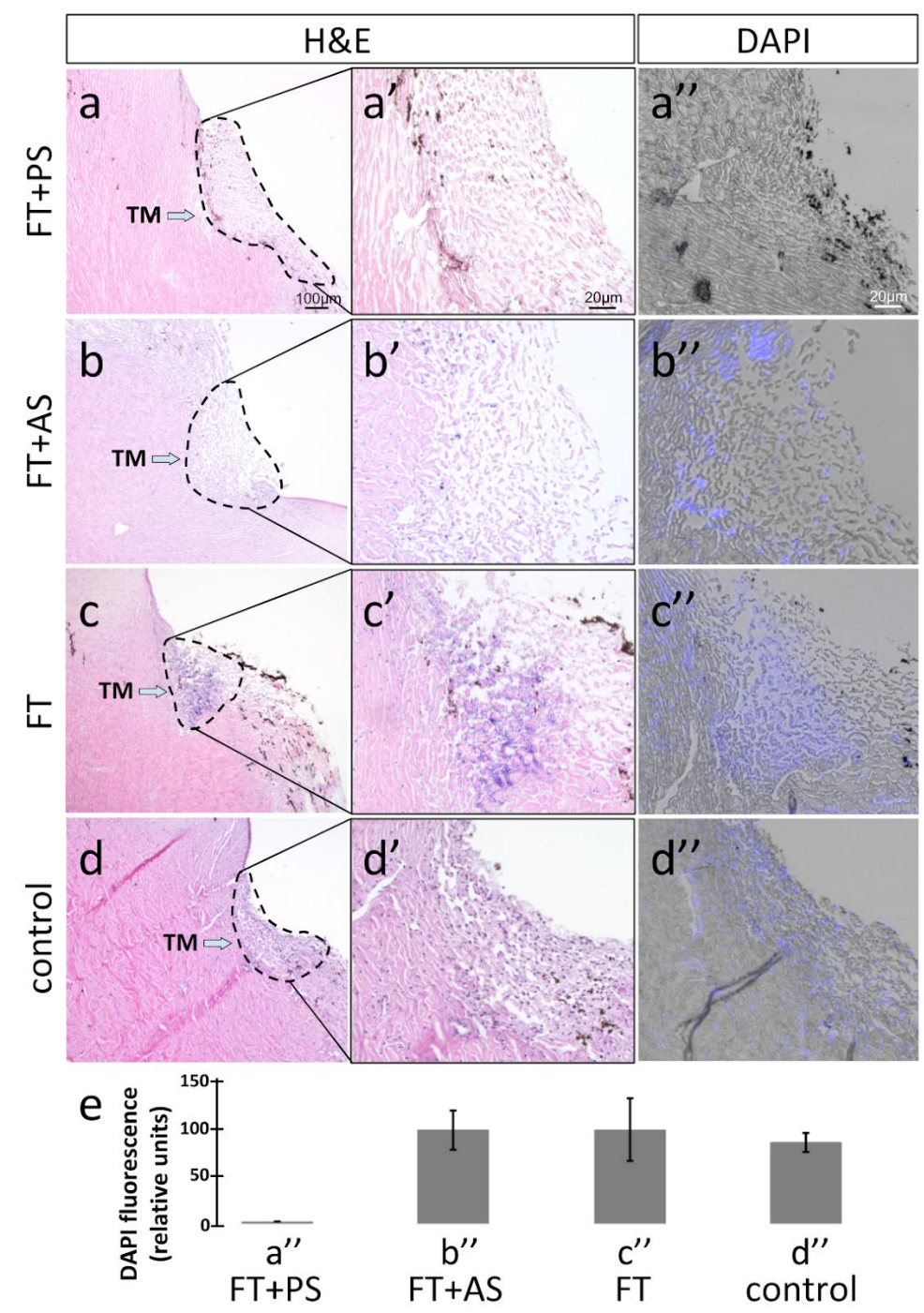

Fig. 1: Left: H\&E staining, middle: magnified view of TM. Right: DAPI + brightfield. (a) Freeze-thaw cycling and perfusion of surfactant (FT+PS) removed visible cells ( $a^{\prime}$ ) and nuclear debris (no blue DAPI staining (a")). (b) Cellularity was reduced after FT and agitation in surfactant (FT+AS, $\left.\left(b^{\prime}\right)\right)$, and nuclear debris was seen in distal TM (b"). (c) FT alone destroyed cells but nuclear material was present in mid and distal TM ( $c^{\prime}$ and $\left.c^{\prime \prime}\right)$. (e) Untreated controls with normal TM cellularity ( $\left.e^{\prime}\right)$ and even, intranuclear DAPI staining pattern ( $\left.e^{\prime \prime}\right)$. DAPI fluorescence was greatly reduced in FT+PS but similar in groups FT+AS $\left(b^{\prime \prime}\right), F T\left(c^{\prime \prime}\right)$, and controls (d"). 
Figure 2: IOP within physiological range throughout perfusion-decellularization

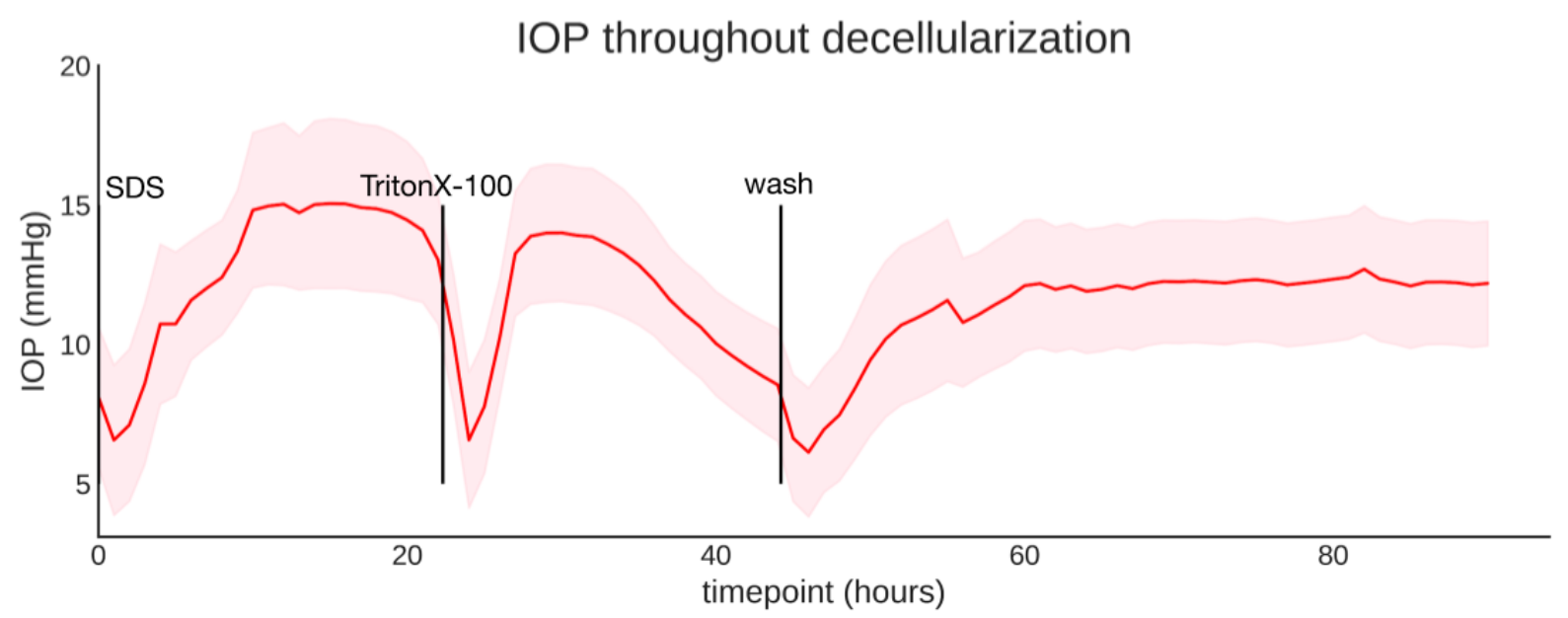

Fig 2: IOP remains within a physiological range throughout perfusion decellularization $(12.27 \pm 0.04 \mathrm{mmHg}, \mathrm{n}=15)$. Brief IOP drops after media exchanges are due to lapse in perfusion during a system restart. Error fields indicate SEM. 
Figure 3: Seeded cells localized to the TM region

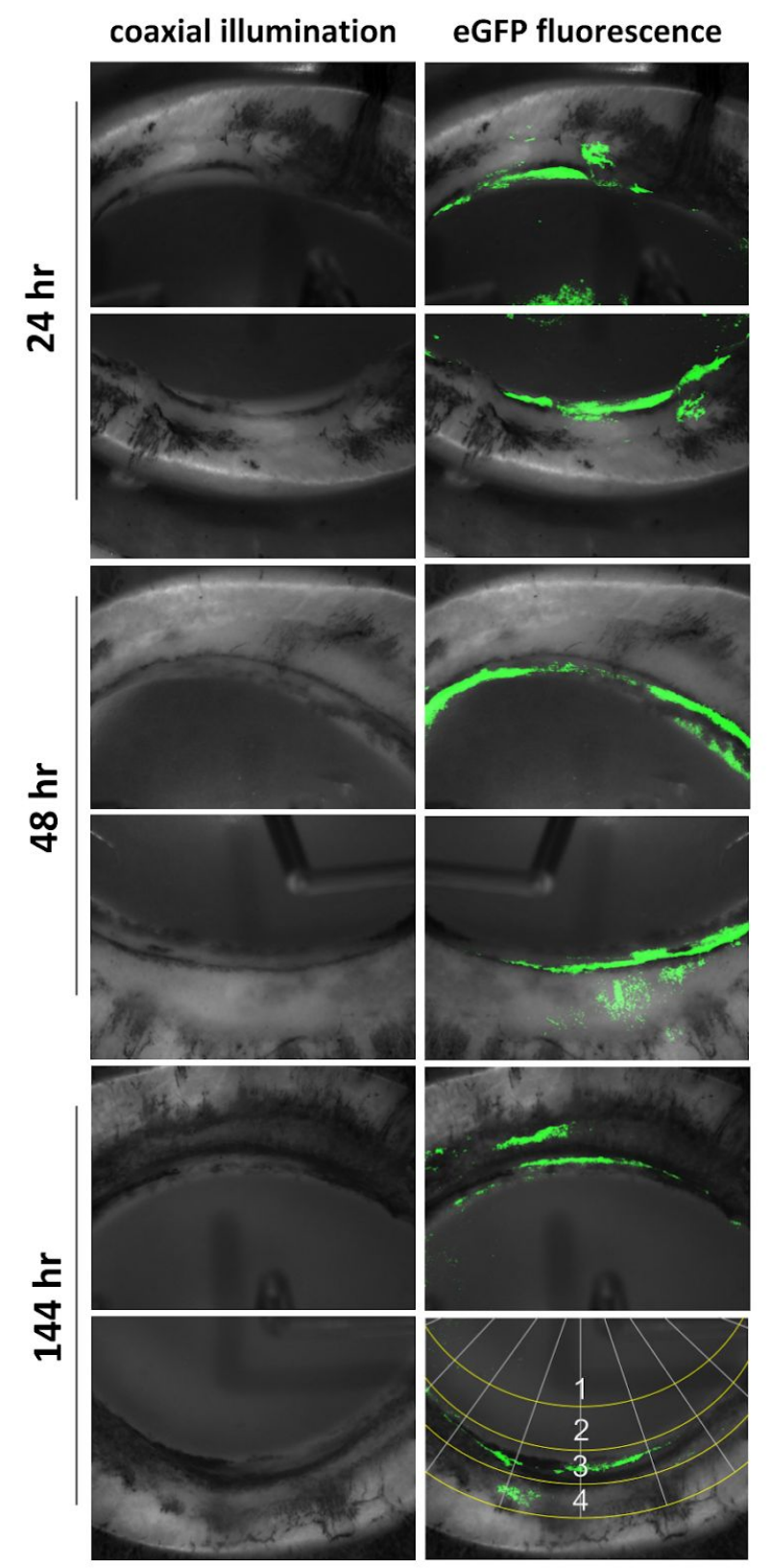

Fig. 3: Seeded cells localized to the TM region. Anterior segment scaffolds seeded with 3 million eGFP CrFK cells were visualized directly through the underside of clear perfusion culture dishes. Left: brightfield view of TM angle, right: merged with eGFP fluorescence. Localization of intensity in fluorescence images was evaluated in the 4 concentric regions shown at the bottom right panel (central cornea (1), peripheral cornea (2), TM (3), and sclera/ciliary body remnant (4).) TM was brighter than all other regions $(90.1 \pm 7.5 \%$ brighter, $p<0.001$.) 
Figure 4: Cell infiltration of the TM increases with time in perfusion culture
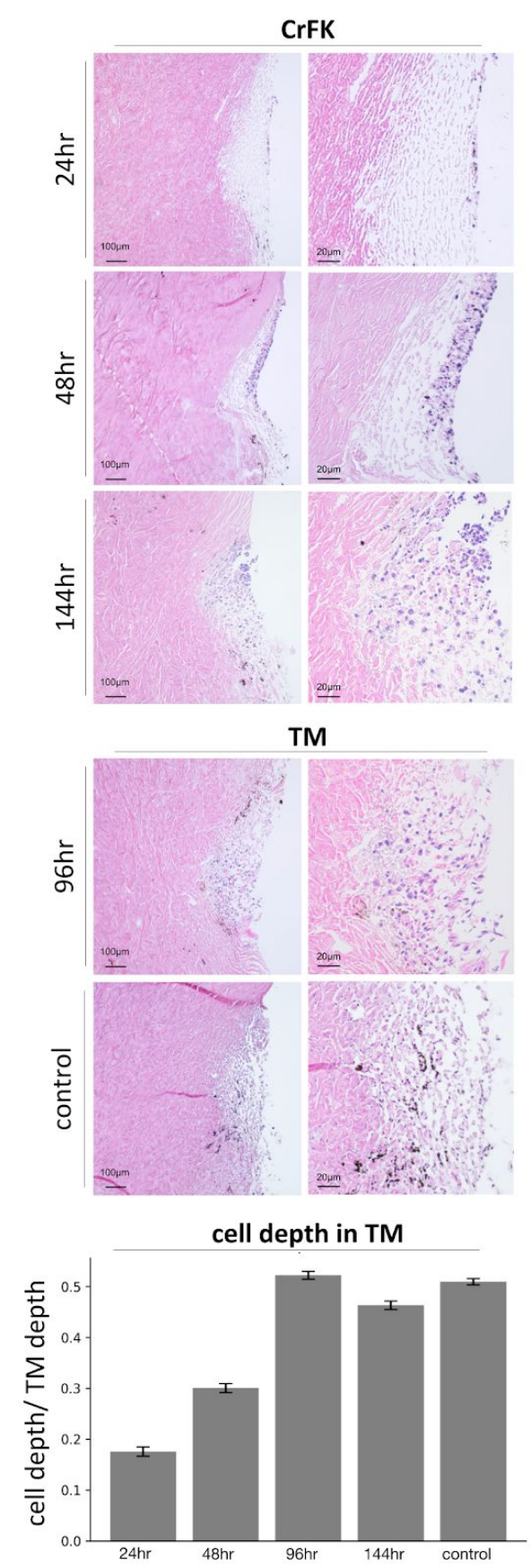

Fig. 4: Cell infiltration of the TM at 24, 48, and 144 hours for cultures reseeded with CrFK cells shown in Fig. 3. Cell infiltration of scaffolds reseeded with porcine TM cells is shown at $96 \mathrm{hrs}$. Cell depth at 24 and 48 hours was significantly different from control $(p<0.001)$ while cell depth at 96 and 144 hours and above was not significantly different $(p=0.19,0.82)$. Error bars indicate SEM. 
Figure 5: IOP of tissue-engineered ex vivo cultures

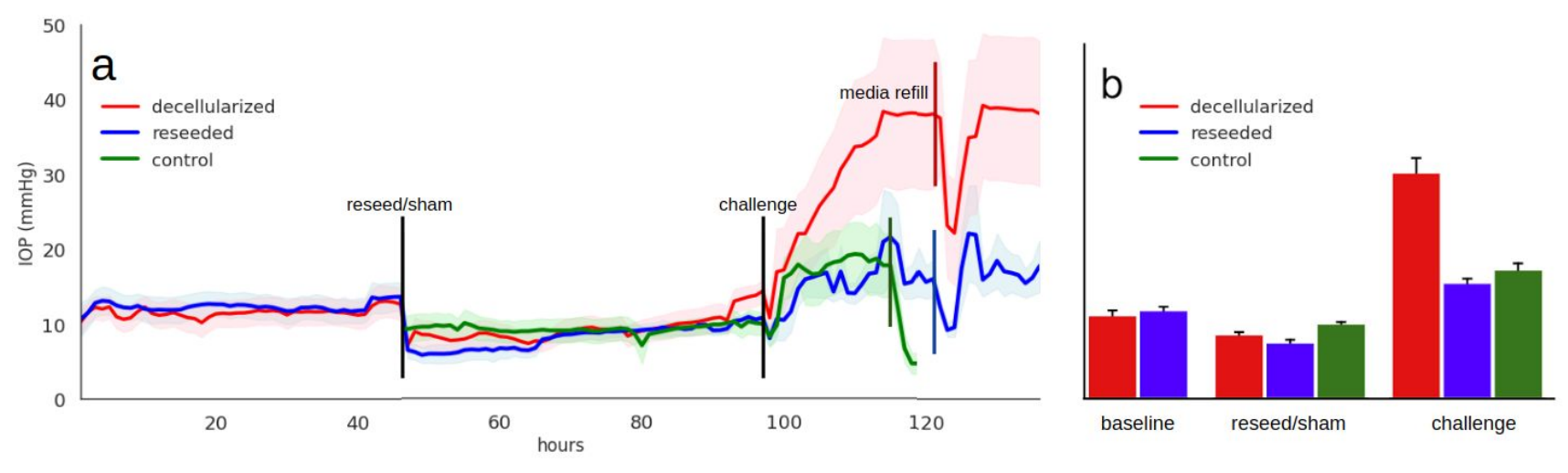

Fig. 5: IOP of tissue-engineered ex vivo cultures. A) Time-series IOP data of decellularized scaffolds, reseeded scaffolds, and normal anterior segment culture controls. B) Summary of IOP at each experimental phase. No significant difference was found between decellularized (D) and reseeded $(R S)$ at baseline $(p=0.40)$ or after cell seeding/sham $(p=0.07)$. After 48 hours, the infusion rate was doubled from $3 \mu \mathrm{L} / \mathrm{min}$ to $6 \mu \mathrm{L} / \mathrm{min}$. Reseeded (blue) eyes maintained IOP within a normal range while IOP in decellularized scaffolds was significantly elevated $(p<$ $0.0001, n=8$ for each). Error fields indicate SEM, ns= not significant, ${ }^{*} p<0.0001$. 


\section{Tables}

Table 1: Cell infiltration of scaffolds

\begin{tabular}{cccccc}
\hline time point & $\mathbf{2 4}$ hours & $\mathbf{4 8}$ hours & $\mathbf{9 6}$ hours & $\mathbf{1 4 4}$ hours & control \\
\hline cell type & CrFK & CrFK & TM & CrFK & TM \\
$\begin{array}{c}\text { depth in } \\
\text { meshwork (\%) }\end{array}$ & $17.5 \pm 0.9^{*}$ & $30.0 \pm 0.9^{*}$ & $52.2 \pm 0.7$ & $49.1 \pm 0.7$ & $50.9 \pm 0.6$ \\
$\begin{array}{c}\text { cellularity } \\
\text { (per section) }\end{array}$ & $9.2 \pm 1.6^{*}$ & $23.1 \pm 4.4^{*}$ & $60.1 \pm 6.4^{*}$ & $60.0 \pm 13.0$ & $100.0 \pm 11.1$
\end{tabular}

Table 1: Cell infiltration of scaffolds. Depth of infiltrated cells is shown relative to the complete depth of the meshwork. Tissue-engineered TM cellularity values at each time-point are normalized to control. Error indicates SEM. Asterisk indicates significant difference from respective control $(p<0.05)$. 